\title{
The use of nanoencapsulation to decrease human skin irritation caused by capsaicinoids
}

\author{
This article was published in the following Dove Press journal: \\ International Journal of Nanomedicine \\ 12 February 2014 \\ Number of times this article has been viewed
}

\author{
Renata $V$ Contri' \\ Luiza A Frank ${ }^{2}$ \\ Moacir Kaiser ${ }^{1}$ \\ Adriana R Pohlmann ${ }^{1,3}$ \\ Silvia S Guterres ${ }^{1,2}$ \\ 'Programa de Pós-Graduação em \\ Ciências Farmacêuticas, ${ }^{2}$ Faculdade \\ de Farmácia, ${ }^{3}$ Instituto de Química, \\ Universidade Federal do Rio Grande \\ do Sul, Porto Alegre, Rio Grande do \\ Sul, Brazil
}

Correspondence: Silvia S Guterres Faculdade de Farmácia, Universidade Federal do Rio Grande do Sul, Av lpiranga, 2752/405 CEP 906/0-000, Porto Alegre, Rio Grande do Sul, Brazil Tel +55 5l 33085500

Fax +555 I 33085247

Email silvia.guterres@ufrgs.br

\begin{abstract}
Capsaicin, a topical analgesic used in the treatment of chronic pain, has irritant properties that frequently interrupt its use. In this work, the effect of nanoencapsulation of the main capsaicinoids (capsaicin and dihydrocapsaicin) on skin irritation was tested in humans. Skin tolerance of a novel vehicle composed of chitosan hydrogel containing nonloaded nanocapsules (CH-NC) was also evaluated. The chitosan hydrogel containing nanoencapsulated capsaicinoids (CH-NC-CP) did not cause skin irritation, as measured by an erythema probe and on a visual scale, while a formulation containing free capsaicinoids (chitosan gel with hydroalcoholic solution [CH-ET-CP]) and a commercially available capsaicinoids formulation caused skin irritation. Thirty-one percent of volunteers reported slight irritation one hour after application of CH-NC-CP, while moderate (46\% [CH-ET-CP] and 23\% [commercial product]) and severe ( $8 \%$ [CH-ET-CP] and $69 \%$ [commercial product]) irritation were described for the formulations containing free capsaicinoids. When $\mathrm{CH}-\mathrm{NC}$ was applied to the skin, erythema was not observed and only $8 \%$ of volunteers felt slight irritation, which demonstrates the utility of the novel vehicle. A complementary in vitro skin permeation study showed that permeation of capsaicinoids through an epidermal human membrane was reduced but not prevented by nanoencapsulation.
\end{abstract}

Keywords: chitosan, nanocapsules, capsaicinoids, skin irritation, skin permeation

\section{Introduction}

Nanoparticles used as drug delivery systems have particle sizes in the range of $10-1,000 \mathrm{~nm}$. Depending on the methods and raw materials used for preparation, different structures can be obtained. Frequently, they are composed of an oily core surrounded by a polymer membrane and are known as nanocapsules. ${ }^{1-3}$ These systems have several advantages, including controlled and sustained release of the drug, leading to a more effective and less toxic treatment than when using conventional options. ${ }^{3,4}$ Regarding cutaneous use, these nanocapsules can act as a reservoir for lipophilic drugs, ${ }^{5}$ modulating their penetration/permeation across the skin by controlling contact of the substances with the stratum corneum. . $^{5,6}$

Recently, nanotechnology has been proposed as a strategy to decrease the irritation and allergenicity of active substances after application to the skin, by controlling the rate of release of the irritant substance ${ }^{7}$ and avoiding direct contact between the substance and the skin. ${ }^{8,9}$ Using the Draize patch test in rabbits, Shah et $\mathrm{l}^{8}$ showed that nanoencapsulation of tretinoin in solid lipid nanoparticles resulted in better skin tolerance when compared with a commercially available cream formulation of tretinoin. The effect of nanoencapsulation with regard to decreasing skin irritation 
after application of retinoic acid was also determined using mouse skin and a visual scale. ${ }^{7}$ Pople et $\mathrm{al}^{9}$ encapsulated tacrolimus into lipid nanoparticles and observed a less irritating effect using the Draize skin patch test in rabbits when compared with a commercial formulation. To the authors' knowledge, the ability of a nanotechnology-based formulation to decrease the skin irritation of a substance has not yet been demonstrated in humans.

Capsaicin (trans-8-methyl-N-vanillyl-6-nonenamide) is a topical analgesic used in the treatment of chronic pain in many diseases, including osteoarthritis, rheumatoid arthritis, diabetic neuropathy, and post-herpetic neuralgia, as well as in the symptomatic treatment of psoriais. ${ }^{10,11}$ Capsaicin is commercially available as a cream formulation, ${ }^{10}$ and more recently, as a high-concentration adhesive. ${ }^{12}$ When in contact with peripheral nerves, capsaicin causes desensitization after a considerable number of applications. ${ }^{10}$ It has irritant properties when applied to the skin, so treatment cannot be given continuously, therefore little or no efficacy is achieved, given that repeated application is necessary for the analgesic action of capsaicin. ${ }^{13,14}$

Development of capsaicin analogs has been proposed in order to obtain the benefits of this substance while avoiding its irritating effect. ${ }^{10}$ Nanoencapsulation represents an alternative strategy for use of capsaicin, while decreasing its currently observed side effects.

Capsaicinoids are found most abundantly in chili peppers (capsaicin and dihydrocapsaicin), ${ }^{10}$ and have been successfully co-nanoencapsulated in acrylic-based nanocapsules. ${ }^{15}$ It has been demonstrated that nanoencapsulation decreases the rate of release of capsaicinoids compared with micellar and hydroalcoholic solutions containing these substances. ${ }^{15}$ A formulation for cutaneous application of capsaicinoids was also obtained by incorporating nanocapsules into a chitosan hydrogel. ${ }^{16}$ The formulation obtained by chitosan hydrogel and nanocapsules has shown controlled release ${ }^{16}$ and good skin adhesion properties. ${ }^{17}$ Chitosan has several applications in drug delivery systems for specific routes of administration, due to the unique cationic character of chitosan. $^{18,19}$

The present study evaluated the effect of nanoencapsulation on the skin irritation process after application of capsaicinoids, and attempted to confirm skin tolerance of a novel vehicle based on chitosan hydrogel and polymeric nanocapsules. Skin permeation studies were also undertaken in an attempt to gain a better understanding of the skin irritation caused by capsaicinoids.

\section{Materials and methods \\ Materials}

Eudragit RS $100^{\circledR}$, polysorbate 80 , and capric/caprylic triglycerides were obtained from Degussa (Darmstadt, Germany), Labsynth (São Paulo, Brazil), and Brasquim (Porto Alegre, Brazil), respectively. Chitosan of medium molecular weight (77\% deacetylated) was purchased from Sigma-Aldrich (São Paulo, Brazil) and lactic acid was sourced from Via Farma (São Paulo, Brazil). Capsaicinoids (containing 58.61\% capsaicin and 33.76\% dihydrocapsaicin) were obtained from Deg (São Paulo, Brazil), while a commercial capsaicinoid cream was obtained from a drugstore in Porto Alegre, Brazil. The commercial cream contained methylparaben, self-emulsifying wax, Vaseline ${ }^{\circledR}$, and deionized water as excipients. Acetone (Rio de Janeiro, Brazil), ethanol (São Paulo, Brazil), and acetonitrile (Fairfield, OH, USA) were of analytical and high pressure liquid chromatography (HPLC) grade.

\section{Manufacture and characterization of formulations \\ Manufacture of nanocapsules}

The nanocapsules were obtained by the interfacial deposition of a preformed polymer method ${ }^{20}$ using Eudragit RS $100^{\circledR}$ as the polymeric wall. The biocompatible polymer selected was a copolymer of poly(ethyl acrylate, methylmethacrylate) containing ammonium quaternary groups. Eudragit RS $100^{\circledR}$ has already been described for creating nanocapsules intended for specific administration routes, including cutaneous, ${ }^{15}$ vaginal, ${ }^{21}$ and ocular. ${ }^{22}$ Capric/ caprylic triglycerides were used to obtain the oily core, polysorbate 80 was used as a stabilizer, and capsaicinoids were used as the nanoencapsulated substances, identical to a previously described manner..$^{15}$ The final theoretical capsaicinoid concentration in the aqueous nanocapsule suspension was $0.5 \mathrm{mg} / \mathrm{mL}(0.29 \mathrm{mg} / \mathrm{mL}$ of capsaicin and $0.17 \mathrm{mg} / \mathrm{mL}$ of dihydrocapsaicin). Similarly, an unloaded nanocapsule formulation was created by omitting the active substances for use as a control in the skin irritation test, as well as to test the skin tolerance of the proposed novel vehicle.

\section{Characterization of nanocapsules}

Prior to their incorporation in the chitosan hydrogel, the nanocapsules were characterized in terms of size, polydispersity, zeta potential, drug content, and encapsulation efficiency. 
The average diameter (D[4.3]) and SPAN (polydispersity) value were determined by laser diffraction (Mastersizer ${ }^{\circledR} 2000$, Malvern Instruments, Malvern, UK), applying the refraction index of Eudragit RS 100 (1.38). The SPAN value was obtained as a ratio between D0.9 (decreased from D0.1) and D0.5, considering D0.9, D0.5, and D0.1, the diameters that correspond to $10 \%, 50 \%$, and $90 \%$ of the particles, respectively, in the size distribution. The aqueous suspensions were added to the equipment sampling apparatus (a small-volume manual sample dispersion unit) containing distilled water until the desired laser obscuration was reached (2\%).

The zeta potential was determined by electrophoretic mobility (Zetasizer ${ }^{\circledR}$ ZEN 3600, Malvern Instruments) after dilution in $\mathrm{NaCl} 10 \mathrm{mM}(1: 500 \mathrm{v} / \mathrm{v})$ aqueous solution.

Drug content and encapsulation efficiency were investigated using an HPLC-UV system (Series 200, PerkinElmer, Waltham, MA, USA) equipped with a $\mathrm{C} 18$ reversed phase column (Merck \& Co, Inc, Whitehouse Station, NJ, USA) at $280 \mathrm{~nm}$, according to a prevalidated methodology. ${ }^{15}$ The mobile phase was composed of a mixture of acetonitrile and water $(70: 30, \mathrm{v} / \mathrm{v})$, with $\mathrm{pH}$ adjusted to $4.5 \mathrm{using}$ acetic acid, running at a flow rate of $0.75 \mathrm{~mL}$ per minute. Samples of the capsaicinoid-loaded nanocapsule suspension were solubilized in acetonitrile (for drug content) or submitted to ultrafiltration-centrifugation (for encapsulation efficiency) (Ultrafree-MC 10 kDa, EMD Millipore, Billerica, MA, USA) before chromatographic analysis.

\section{Construction of hydrogels \\ Chitosan hydrogels containing nanocapsules}

The two formulations used in this work were obtained by dispersion of chitosan in the aqueous nanocapsule suspension, followed by addition of lactic acid and manual mixing of the formulation. When the capsaicinoid-loaded nanocapsule suspension was used for the hydrogel production, the formulation was labeled CH-NC-CP while it was labeled CH-NC when the unloaded nanocapsule suspension was used.

\section{Chitosan hydrogels containing hydroalcoholic} solution

Two other chitosan gels were obtained for the in vivo skin irritation study. The first corresponded to the chitosan hydrogel containing capsaicinoids in the free or nonencapsulated form (CH-ET-CP). The chitosan was dispersed in a hydroalcoholic solution ( $30 \%$ ethanol) containing capsaicinoids at a concentration of $0.5 \mathrm{mg} / \mathrm{mL}$, ie, the same as that found in the nanocapsule suspension. The amount of ethanol in the hydroalcoholic solution was the lowest amount capable of completely solubilizing the capsaicinoids. The addition of lactic acid and manual mixing proceeded as mentioned earlier. The second formulation was the chitosan dispersion in a blank (without capsaicinoids) hydroalcoholic solution (30\% ethanol) known as CH-ET, which was used to confirm that the irritation effect was due to the capsaicinoids. Table 1 summarizes the chitosan hydrogels developed in this work and their components.

\section{Characterization of semisolid formulations}

The hydrogels shown in Table 1 and the commercial capsaicinoid formulation were characterized in terms of $\mathrm{pH}$, viscosity and capsaicinoid content. The $\mathrm{pH}$ value was obtained by potentiometry (B474, Micronal S.A., Sao Paolo, Brazil), after dilution of the formulation in ultrapure water $(1: 10 \mathrm{w}: \mathrm{v})$. The viscosity was determined as a function of the shear rate by means of rotary viscosimetry (LV-DV-II+Pro, Brookfield Engineering Laboratories, Middleboro, MA, USA), using spindle SC4-25 at $25^{\circ} \mathrm{C} \pm 1{ }^{\circ} \mathrm{C}$. The capsaicinoid content in the CH-NC-CP, CH-ET-CP, and commercial formulation was determined by a validated HPLC-ultraviolet methodology. ${ }^{15}$ The capsaicinoids were extracted from the formulations with acetonitrile ( 2 hours under mixing at $37^{\circ} \mathrm{C}$ ) and quantified as described earlier for the nanocapsule suspensions.

The formulations containing nanocapsules were also evaluated in terms of their size distribution by laser diffraction

Table I Composition of hydrogels

\begin{tabular}{lllll}
\hline Formulations & \multicolumn{2}{l}{ Components } & & \\
\cline { 2 - 5 } & Chitosan (2.5\%) & Lactic acid (1\%) & $\begin{array}{l}\text { Nanocapsule } \\
\text { suspension }\end{array}$ & $\begin{array}{l}\text { Hydroalcoholic } \\
\text { solution }\end{array}$ \\
\hline $\mathrm{CH}-\mathrm{NC}-\mathrm{CP}$ & $\mathrm{x}$ & $\mathrm{x}$ & $\mathrm{x}$ & $\mathrm{x}$ \\
$\mathrm{CH}-\mathrm{NC}$ & $\mathrm{x}$ & $\mathrm{x}$ & $\mathrm{x}$ & $\mathrm{x}$ \\
$\mathrm{CH}-\mathrm{ET}-\mathrm{CP}$ & $\mathrm{x}$ & $\mathrm{x}$ & & $\mathrm{x}$ \\
$\mathrm{CH}-\mathrm{ET}$ & $\mathrm{x}$ & $\mathrm{x}$ & & $\mathrm{x}$ \\
\hline
\end{tabular}

Note: $x$, used in the formulation preparation.

Abbreviations: $\mathrm{CH}$, chitosan gel; NC, nanocapsules; $\mathrm{CP}$, capsaicinoids; $\mathrm{ET}$, ethanolic solution. 
(Mastersizer $^{\circledR}$ 2000, Malvern Instruments, Malvern, UK), applying the refraction index of Eudragit RS 100 (1.38) in order to confirm the presence of the nanoparticles. The measurements were performed as described for the nanocapsule aqueous suspension.

Except for viscosity, which was measured once, the properties of the semisolid formulations were determined in triplicate batches for the chitosan hydrogels and in triplicate measurements of the commercial formulation.

\section{In vivo skin irritation}

The skin irritation study for the test formulation $(\mathrm{CH}-$ NC-CP) and controls (CH-NC, CH-ET-CP, CH-ET, and the commercial product) was approved by the university ethics on research committee (protocol number 21520). After obtaining informed consent, the volunteers $(n=13$, males and females aged 18-45 years) washed their arms with neutral soap, and three circles $30 \mathrm{~mm}$ in diameter were drawn on each arm with a cast mold. Using a plastic spatula, $150 \mathrm{mg}$ of each formulation (CH-NC-CP, CH-ET-CP, and the commercial product) were applied to each circle, giving a final capsaicinoid concentration of $0.01 \mathrm{mg}$ per $\mathrm{cm}^{2}$ of skin area. The formulation was left in contact with the skin for 30 minutes, and then removed using running water and wipers. One circle on the arm corresponded to a control area without application of any formulation.

Skin irritation was evaluated by double-blind evaluation of skin erythema formation using an electronic probe (Mexameter $^{\circledR}$, CK Electronic, Cologne, Germany) and an arbitrary visual scale from 0 to $5(0$, redness; 1 , slight red points; 2, bigger red points; 3 , concrete regions of redness; 4 , redness encompassing the whole circle where the formulation was applied; 5 , redness extending beyond the drawn circle). A sensory study, in which the volunteers reported if they were feeling no, slight, moderate, or severe skin irritation symptoms (pruritus, burning, or pain) was conducted simultaneously. Data acquisition was done before and 30,60,90, 120, and 180 minutes after application of the formulations. At each time point, a visual analysis was performed followed immediately by probe measurement. Skin transepidermal water loss and $\mathrm{pH}$ values were also studied as indicators of skin irritation. ${ }^{23}$ These measurements (using a Tewameter TM30M ${ }^{\circledR}$ and Skin-pH-Meter ${ }^{\circledR}$, CK Electronic) were performed before and 60 and 180 minutes after application of the test formulations.

\section{In vitro skin permeation}

The skin permeation study was performed using an automated Franz cell (Microette Plus Multi-Group ${ }^{\circledR}$, Hanson Research
Corporation, Chatsworth, PA, USA), with heat-separated epidermis as the membrane. Human skin was selected because of the high likelihood of an in vitro-in vivo correlation. ${ }^{6}$ The human skin samples were obtained following plastic surgery, after securing informed consent from the patient. Intact skin (with an intact barrier) was used for the in vitro skin permeation experiment in order to allow a comparison with the skin irritation observed in vivo. Therefore, the proposed permeation study only provides information in a situation where the skin serves as an effective barrier, which may not always be the case, depending on the pathology associated with the pain treated using topical capsaicin. After receiving the skin samples, the adipose tissue was removed and the outer skin layer was cleaned with sodium lauryl sulfate solution $1 \%(\mathrm{w} / \mathrm{v})$ followed by ultrapure water. The skin was cut into circles and placed in beakers containing heated water $\left(60^{\circ} \mathrm{C}\right)$ for 60 seconds. The epidermis was carefully removed and, after part of the water was evaporated, the remaining membrane was cut into smaller circles approximately $3 \mathrm{~cm}$ in diameter. The heat-separated epidermis had a thickness of $0.08-0.12 \mathrm{~mm}$ as determined using a dial thickness gauge $7301{ }^{\circledR}$ equipment (Mitutoyo).

The test formulation (CH-NC-CP) and control formulations (CH-ET-CP and the commercial product) were applied to the surface of the epidermal membrane $(495 \pm 12 \mathrm{mg}$, skin area $1.76 \mathrm{~cm}^{2}$, following the infinite dose principle, $\mathrm{n}=6$ ). Next $1.2 \mathrm{~mL}$ samples were collected automatically from the receptor medium, comprising $7 \mathrm{~mL}$ of a mixture of ethanol and ultrapure water $(30: 70, \mathrm{v} / \mathrm{v})$ at $1.5,4,8,12,18,24$, and 30 hours after application. Each cell unit was kept under mixing at $32^{\circ} \mathrm{C}$. The amount of capsaicinoid in each collected sample was determined using previously validated HPLC-UV methodology ${ }^{15}$ for determination of capsaicinoid content. In addition, linearity of the method in the receptor medium was ensured before the experiments. Considering a hypothetical situation where the entire amount of capsaicinoid applied permeated the skin, the capsaicinoid concentration in the receptor fluid would be approximately $36 \mu \mathrm{g} / \mathrm{mL}$. Given that the saturated capsaicinoid concentration in the same fluid is $860 \mu \mathrm{g} / \mathrm{mL}$, ie, more than ten times higher than the hypothetical final concentration in the receptor compartment, the sink condition is confirmed. We observed no interference from the epidermal sheet, with the same retention times as the drugs in the chromatograms, confirming the specificity of the method.

The data were analyzed as described in a previous study of skin permeation by capsaicin, ${ }^{11}$ ie, using linear regression of the cumulative amount of capsaicinoid permeated through an epidermis surface unit versus time in the steady-state range. 
Flux was determined by the slope of the regression lines and lag time was determined from the $\mathrm{x}$-intercept values of the regression lines.

\section{Statistical analyses}

The statistical analysis was performed by analysis of variance, followed by Dunnett's test using SigmaStat version 3.5 when a significant difference was detected $(\alpha=0.05)$.

\section{Results and discussion Characteristics of nanocapsules}

The aqueous nanocapsule suspensions had an average diameter (D[4.3]) of $142 \pm 4 \mathrm{~nm}$ (blank nanocapsules) and $138 \pm 2 \mathrm{~nm}$ (loaded nanocapsules). The SPAN values obtained were around $1(1.03 \pm 012$ [blank nanocapsules] and $1.12 \pm 0.13$ [loaded nanocapsules]), indicating good homogeneity of particle size, since the difference between D0.9 and D0.1 is similar to the value of D0.5, which is the size corresponding to half of the size distribution graph. Regarding surface properties, the zeta potential was measured at $+9.13 \pm 0.65 \mathrm{mV}$. The Eudragit RS $100^{\circledR}$ polymer has ammonium quaternary groups which give a positive value to the zeta potential of the nanocapsules. The drug content determined for the capsaicinoid-loaded nanocapsules was $0.49 \pm 0.01 \mathrm{mg} / \mathrm{mL}$ (including both capsaicin and dihydrocapsaicin), while the encapsulation efficiency was close to $100 \%$ (ie, $99.8 \%$ ), probably due to the strong affinity between the capsaicinoids and the oily core of the nanocapsules. Therefore, the aqueous nanocapsule suspensions (unloaded and loaded) had adequate characteristics and were suitable for incorporation into a chitosan gel, leading to a final formulation for cutaneous use.

\section{Characteristics of final formulations}

Hydrogel was the pharmaceutical formulation chosen for delivering the nanocapsules to the skin. The hydrogelforming polymer selected was chitosan, due to its bioadhesion and film-forming properties. ${ }^{24}$ Incorporation of polymeric nanocapsules in a chitosan hydrogel has been previously proposed by our research group, ${ }^{16}$ and the formulation has shown controlled release properties. Furthermore, the abovementioned formulation has been shown to have good skin adhesion, due to the combined effect of the nanocapsules and chitosan. ${ }^{17}$

All the chitosan hydrogels had $\mathrm{pH}$ values close to 4.5 (4.3 \pm 0.2 for $\mathrm{CH}-\mathrm{NC}-\mathrm{CP}, 4.4 \pm 0.1$ for $\mathrm{CH}-\mathrm{NC}, 4.4 \pm 0.2$ for $\mathrm{CH}-$ ETN-CP, and $4.4 \pm 0.2$ for CH-ETN) such that no significant differences were observed between the formulations. Neither the presence of the nanocapsules nor the capsaicinoids led to differences in the $\mathrm{pH}$ values. As previously described, ${ }^{16}$ these values are low due to the presence of lactic acid, but suitable for cutaneous application, given that the skin surface is acidic. ${ }^{25}$ The commercial formulation, on the other hand, had a neutral $\mathrm{pH}$ value of $7.1 \pm 0.1$.

Figure 1 shows viscosity as a function of the shear rate applied to the semisolid formulations. Since a wide difference was observed among the formulations, it was not possible to submit all of them to the same shear rate range. Therefore, they were separated into different graphs. Figure 1A shows viscosity as a function of shear rate for the chitosan hydrogels containing the nanocapsule suspension (CH-NC-CP and $\mathrm{CH}-\mathrm{NC}$ ). No difference was observed when the capsaicinoids were encapsulated within the nanoparticles. Figure 1B

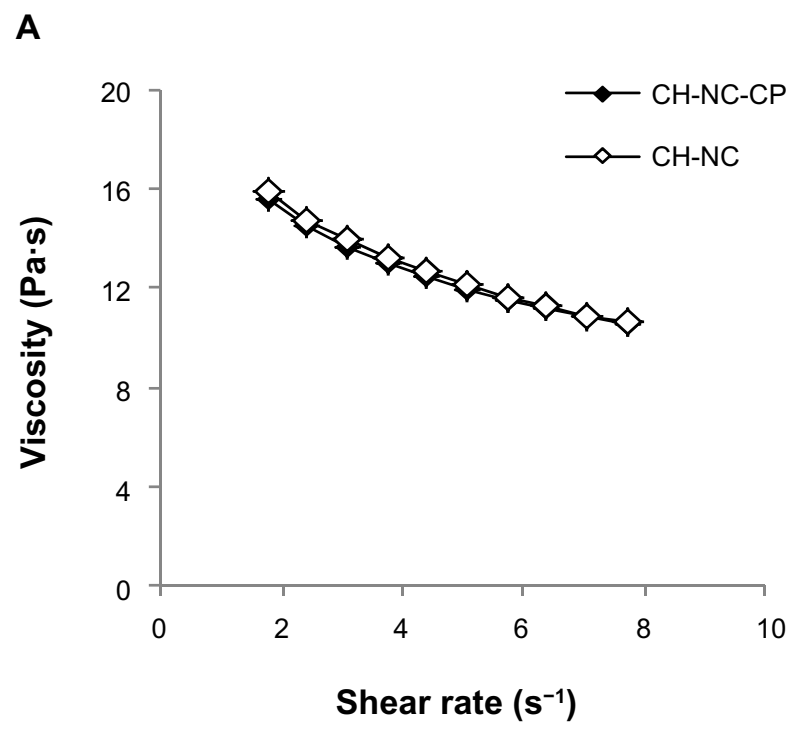

B

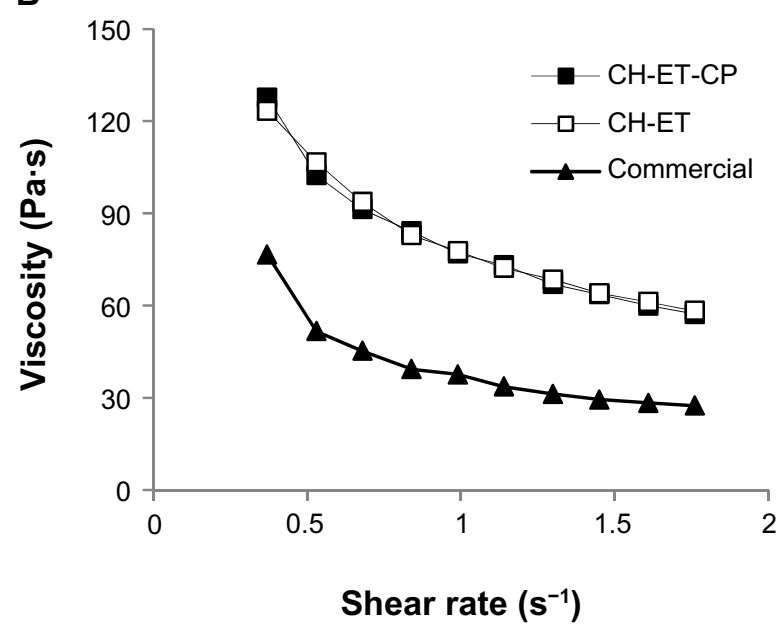

Figure I Viscosity as a function of shear rate of (A) chitosan hydrogels containing nanocapsules and (B) chitosan hydrogels containing ethanol and a commercial formulation of capsaicinoids.

Abbreviations: $\mathrm{CH}$, chitosan gel; NC, nanocapsules; CP, capsaicinoids; $\mathrm{ET}$, ethanolic solution; Commercial, commercial formulation. 
shows viscosity as a function of shear rate for the hydrogels containing hydroalcoholic solution (CH-ETN-CP and $\mathrm{CH}-$ ETN) and for the commercial capsaicinoid formulation. Similarly, no difference was observed due to the presence of capsaicinoids in the gels containing ethanol.

Comparing all formulations in terms of viscosity, it is noticeable that the chitosan hydrogels containing nanocapsules had the lowest viscosity (16 Pascal seconds at a shear rate of 1.76 per second), which was also observed visually. It has been shown previously that polymeric nanocapsules increase the viscosity of a chitosan gel, when compared with a gel containing only chitosan, water, and lactic acid. ${ }^{14}$ Therefore, the lowest viscosity observed in the present work, when compared with the other formulations, is not related to the presence of the nanocapsules. The chitosan hydrogels containing hydroalcoholic solution showed higher viscosity (58 Pascal seconds at a shear rate of 1.76 per second), which might be explained by interactions between the chitosan chains and ethanol, such as hydrogen bonds, approaching the chains and increasing the viscosity of the system. The commercial formulation showed intermediate viscosity (27 Pascal seconds at a shear rate of 1.76 per second). Determination of viscosity is extremely important because it might influence skin permeation, especially when the infinite dose method is used. ${ }^{26}$

The capsaicinoid (capsaicin + dihydrocapsaicin) content in the formulations, determined after the extraction process, was $0.43 \pm 0.04 \mathrm{mg} / \mathrm{g}$ for $\mathrm{CH}-\mathrm{NC}-\mathrm{CP}, 0.41 \pm 0.04 \mathrm{mg} / \mathrm{g}$ for CH-ET-CP, and $0.44 \pm 0.01 \mathrm{mg} / \mathrm{g}$ for the commercial product, with no significant difference between the formulations. This result was a prerequisite for the skin studies that followed. Given that capsaicin and dihydrocapsaicin (the major capsaicinoids) have irritant properties, ${ }^{10}$ it was important to determine the content of each and obtain a sum of the two capsaicinoids, instead of considering only the amount of capsaicin.

The two formulations containing nanocapsules $(\mathrm{CH}-$ NC-CP and CH-NC) were analyzed in terms of size distribution by laser diffraction using the refraction index of the polymer in the nanocapsule shell (Figure 2). A nanometric size distribution was observed, very similar to the size distribution verified for the nanocapsules in aqueous suspension (data not shown), indicating no irreversible aggregation or changes in particle size.

\section{Skin irritation in vivo}

Different approaches were taken in order to determine skin irritation, ie, measurement and visual determination of

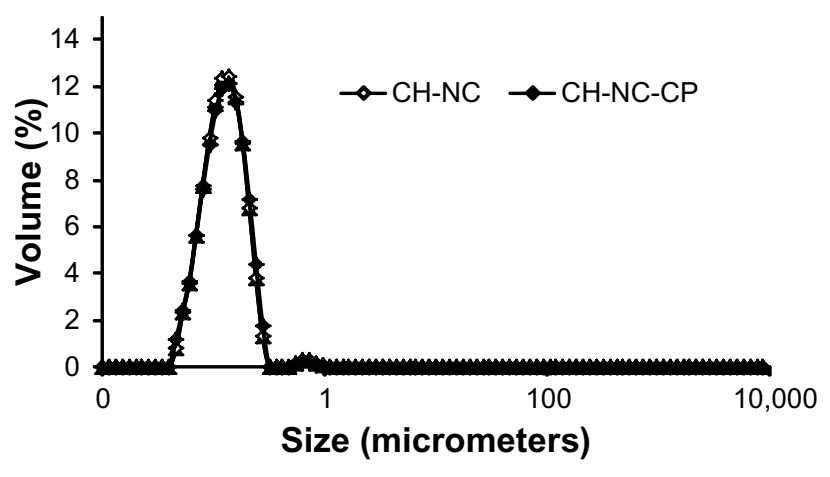

Figure 2 Size distribution analyses of chitosan hydrogels containing nanocapsules. Abbreviations: $\mathrm{CH}$, chitosan gel; $\mathrm{NC}$, nanocapsules; $\mathrm{CP}$, capsaicinoids.

erythema, measurement of other skin properties $(\mathrm{pH}$ and transepidermal water loss), and sensory analysis.

\section{Measurement and visual determination of erythema}

The final erythema value, measured by the Mexameter probe (CK Electronic), was determined as the ratio between measurements at the predetermined time and time zero, corresponding to the data obtained before application of the formulation. This data correction was performed because the time zero measurements for erythema differed considerably between the volunteers. Graphs of the final erythema value as a function of time are shown in Figure 3. The formulations were compared with the untreated control area at each time point, and when significantly $(P<0.05)$ higher erythema values were obtained, the formulation was considered to have caused skin erythema.

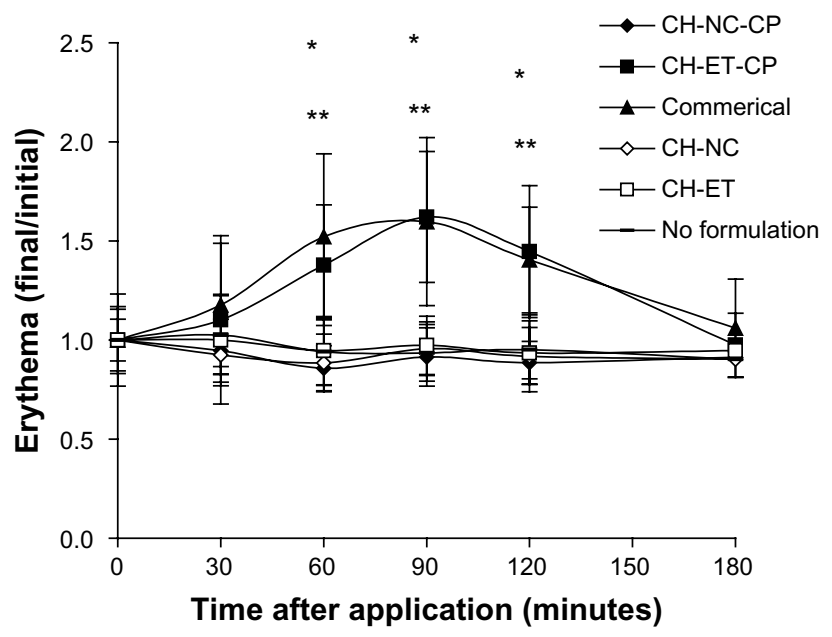

Figure 3 Skin erythema measured by electronic probe. The results indicate a ratio between the initial values (before application of formulation) and the final value at every measuring time point.

Notes: Significant differences observed $(P<0.05)$ : ${ }^{*} \mathrm{CH}-\mathrm{ET}-\mathrm{CP}$ versus no formulation; ***ommercial formulation versus no formulation.

Abbreviations: $\mathrm{CH}$, chitosan gel; NC, nanocapsules; $\mathrm{CP}$, capsaicinoids; $\mathrm{ET}$, ethanolic solution; Commercial, commercial formulation. 
By measurement with the probe, the chitosan hydrogel containing nanocapsules (CH-NC-CP) did not cause erythema at any time point considered. The CH-ET-CP and commercial formulations caused erythema at time points 60 (1.38 \pm 0.30 for CH-ET-CP and $1.52 \pm 0.42$ for the commercial product), 90 (1.62 \pm 0.33 for CH-ET-CP and $1.60 \pm 0.42$ for the commercial product), and 120 minutes $(1.45 \pm 0.33$ for $\mathrm{CH}$ ET-CP and $1.40 \pm 0.27$ for the commercial product). At the time point when the higher difference was observed between the nanostructured formulation and the controls ( 90 minutes), CH-ET-CP and the commercial product reached values of around 1.6, indicating almost double the amount of erythema measured for the same skin area before topical application, while $\mathrm{CH}-\mathrm{NC}-\mathrm{CP}$ showed a value of around 1 , indicating no erythema formation.

After removal of the formulations (time point 30 minutes), no erythema were detected for any of the products, indicating that the erythema observed for the controls (CH-ET-CP and the commercial product) took some time to start or to be measurable by the probe. Similarly, after 180 minutes, no erythema was detected for any formulation, indicating that the erythema disappeared or became unmeasurable after the time point considered.

Analyzing the formulations that did not contain capsaicinoids, it was observed that the chitosan hydrogel containing unloaded nanocapsules (CH-NC) did not cause erythema at any time point after removal. Similarly, the chitosan gel containing ethanolic solution (CH-ET) did not cause skin erythema. These results are important to demonstrate that the irritation caused by CH-ET-CP was due to the capsaicinoids and not due to the presence of ethanol at a concentration of $30 \%$, and also that the vehicle proposed in the present work, composed of chitosan and nanocapsules, is well tolerated by the skin.

Erythema was also evaluated by visual determination of redness. From the values attributed by the evaluator for all volunteers, the means \pm standard deviations are shown in Figure 4. As in Figure 3, the formulations were compared with the skin area where no formulation was applied at each time point. When significantly $(P<0.05)$ higher values were obtained, it was considered that the formulations caused skin erythema.

Figure 4 is in partial agreement with the results found by the probe. On the visual scale, the test formulation $\mathrm{CH}-$ NC-CP did not cause skin irritation. On the other hand, formulations containing the free drugs caused erythema at all time points studied, ie, 30 minutes $(0.88 \pm 0.74$ for CH-ET-CP and $1.77 \pm 1.22$ for the commercial product),

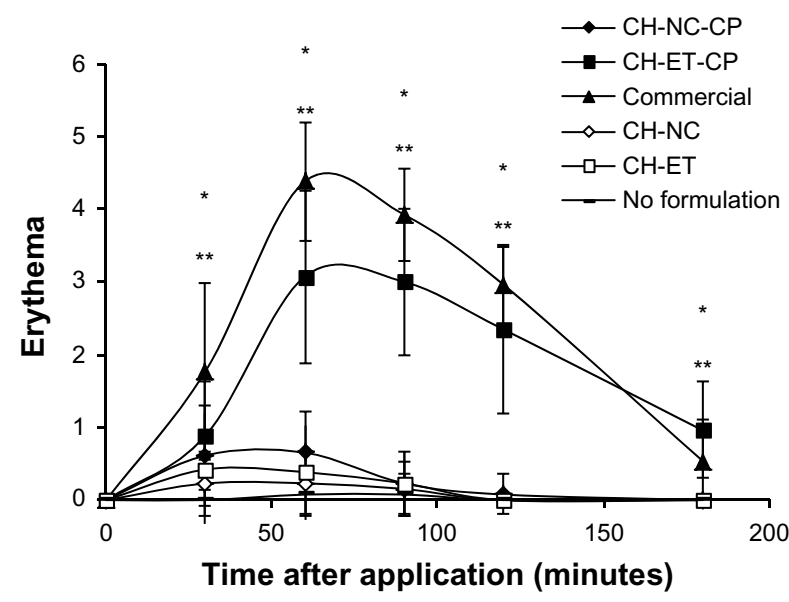

Figure 4 Skin erythema measured by a visual scale.

Notes: Significant differences observed $(P<0.05):{ }^{*} \mathrm{CH}-\mathrm{ET}-\mathrm{CP}$ versus no formulation; **commercial formulation versus no formulation.

Abbreviations: $\mathrm{CH}$, chitosan gel; $\mathrm{NC}$, nanocapsules; $\mathrm{CP}$, capsaicinoids; $\mathrm{ET}$, ethanolic solution; Commercial, commercial formulation.

60 minutes $(3.08 \pm 1.19$ for CH-ET-CP and $4.38 \pm 0.82$ for the commercial product), 90 minutes ( $3.00 \pm 1.00$ for CH-ET-CP and $3.92 \pm 0.64$ for the commercial product), 120 minutes (2.35 \pm 1.14 for CH-ET-CP and $2.96 \pm 0.56$ for the commercial product), and 180 minutes $(0.96 \pm 0.66$ for CH-ET-CP and $0.54 \pm 0.56$ for the commercial product). These results indicate that, at 30 and 180 minutes, the erythema caused by the free drug controls was present, although it was not high enough to be measured by the probe.

It is important to note that CH-ET did not cause erythema during the entire study period. This confirms that the skin irritation observed when CH-ET-CP was applied to the skin was due to the presence of capsaicinoids, and not ethanol, as previously mentioned. Also, the chitosan hydrogel containing unloaded nanocapsules (CH-NC) did not cause erythema at any time point after formulation removal.

Figure 5 shows a photograph obtained from the arm of a volunteer at 90 minutes after application of the formulation. This time point was identified as the time when the greatest differences were observed between the formulations. It is noticeable in the photograph that both CH-ET-CP and the commercial formulation caused erythema, while $\mathrm{CH}-\mathrm{NC}-\mathrm{CP}$, $\mathrm{CH}-\mathrm{NC}$, and $\mathrm{CH}-\mathrm{ET}$ did not.

\section{Skin $\mathrm{pH}$ and transepidermal water loss}

In addition to the skin erythema measurements, skin $\mathrm{pH}$ and water loss values were determined as the ratio between the measurement at the time point considered and the measurement at time zero, corresponding to the data obtained before application of the formulation. With regard to determination of erythema, the time zero measurement differed 


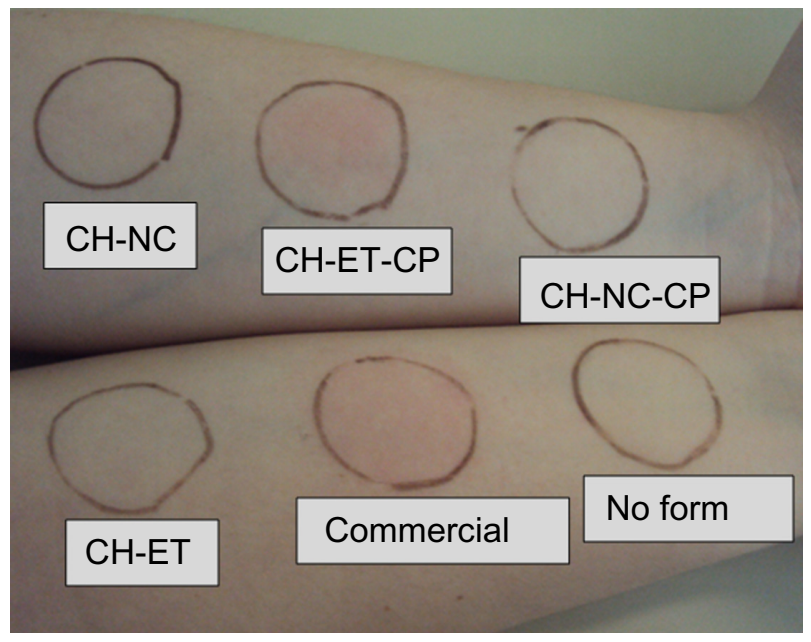

Figure 5 Arm of a volunteer 90 minutes after application of the formulations. The photograph is representative of the entire experiment.

Abbreviations: $\mathrm{CH}$, chitosan gel; NC, nanocapsules; $\mathrm{CP}$, capsaicinoids; $\mathrm{ET}$, ethanolic solution; Commercial, commercial formulation.

considerably between volunteers, so the correction allowed standardization of the values. The formulations were statistically compared with the area where no formulation was applied for each time point.

The $\mathrm{pH}$ (final/initial) and water loss (final/initial) values, as a function of the time, are shown in Figure 6. Compared with the area without application of formulation, no significantly different values were observed for both cases, indicating no changes in $\mathrm{pH}$ or water loss values at 60 and 180 minutes after the formulations were applied to the skin. Therefore, neither the $\mathrm{pH}$ nor the transepidermal water loss values indicated skin irritation. Therefore, only erythema measured by the probe and on the visual scale was used in order to study the human skin irritation caused by capsaicinoids.

Regarding the chitosan gel containing unloaded nanocapsules, no changes in $\mathrm{pH}$ skin values were expected, since the lactic acid used to obtain the chitosan gel has a similar $\mathrm{pKa}$ to that of fatty acids on the skin surface. On the other hand, the transepidermal water loss could have changed due to an occlusive action of the chitosan film. However, this situation was not observed.

\section{Sensory analyses of skin irritation}

The sensory analysis was important to correlate the feeling of irritation with the erythema detected by the probe. Skin irritation includes pruritus, pain, or burning sensation, and not only development of redness. ${ }^{27}$ It is important to analyze the sensation of skin irritation because it is not always well correlated with objective determinations of skin irritation. ${ }^{28}$ The percentages of
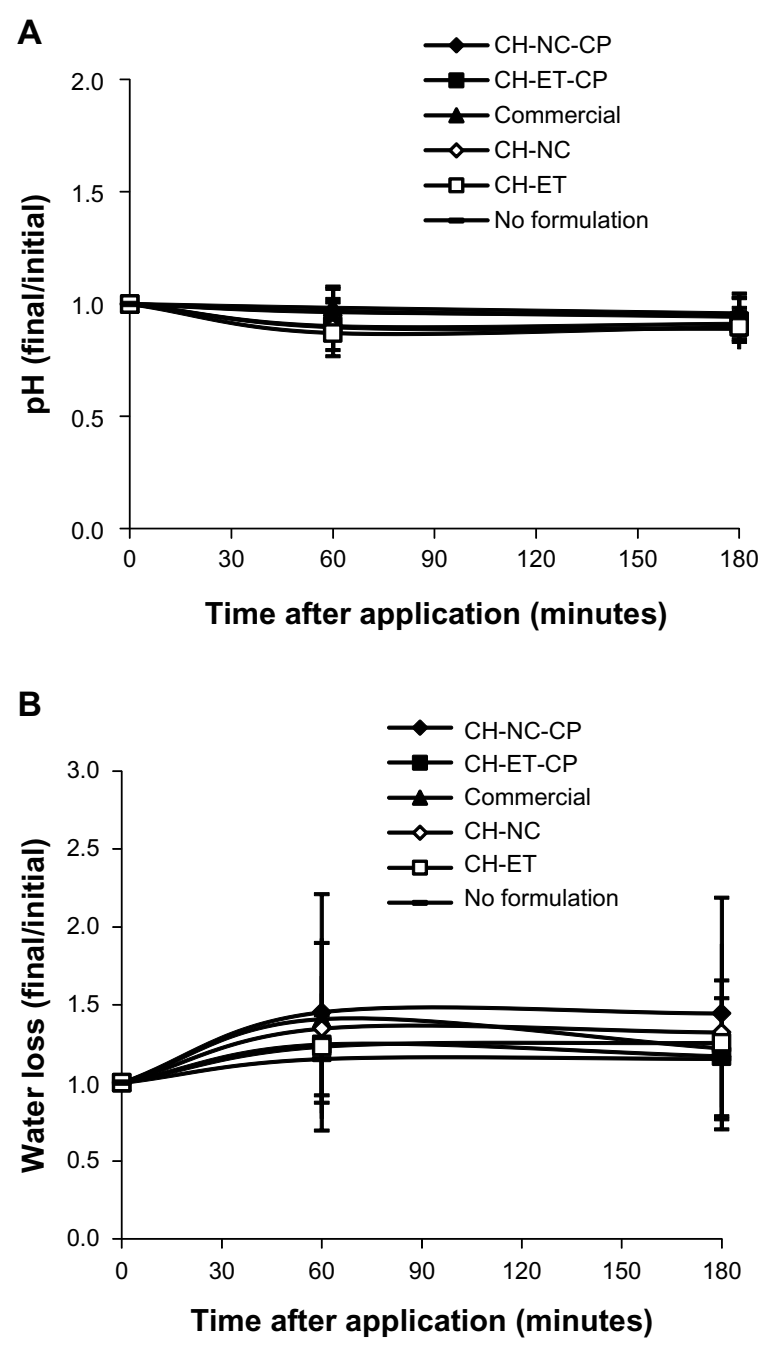

Figure 6 Skin $\mathrm{pH}(\mathbf{A})$ and transepidermal water loss (B) measured by electronic probe. The results indicate a ratio between the initial values (before application of formulation) and the final value at every measuring time point.

Note: No significant differences $(P<0.05)$ are observed between areas with and without application of the formulations.

Abbreviations: $\mathrm{CH}$, chitosan gel; $\mathrm{NC}$, nanocapsules; $\mathrm{CP}$, capsaicinoids; $\mathrm{ET}$, ethanolic solution; Commercial, commercial formulation.

volunteers who felt slight, moderate, or severe irritation at each time point for each formulation are shown in Table 2.

After application of formulation CH-ET-CP, slight irritation was described by the volunteers, moderate irritation after $30(8 \%), 60(45 \%), 90(8 \%)$, and 120 minutes $(15 \%)$, and severe irritation after $60(8 \%)$ and $90(8 \%)$ minutes. Almost $40 \%$ of the volunteers could still feel skin irritation 3 hours after application of CH-ET-CP, indicating that the irritation was persistent. The vehicle obtained with ethanol without capsaicinoids (CH-ET) led only to slight irritation at 30, 60, and 90 minutes, and was felt by a minority of volunteers $(23 \%$, $15 \%$, and $8 \%$, respectively). Once again, this demonstrates that the ethanol in the formulation is not responsible for the skin irritation observed when CH-ET-CP was applied. 
Table 2 Sensory analyses of skin irritation

\begin{tabular}{llllll}
\hline Formulations and & \multicolumn{5}{l}{ Time (minutes) } \\
\cline { 2 - 6 } degree of irritation & $\mathbf{3 0}$ & $\mathbf{6 0}$ & $\mathbf{9 0}$ & $\mathbf{1 2 0}$ & $\mathbf{1 8 0}$ \\
\hline CH-NC-CP & & & & & \\
Negligible & 69 & 69 & 92 & 100 & 100 \\
Slight irritation & 23 & 31 & 8 & 0 & 0 \\
Moderate irritation & 8 & 0 & 0 & 0 & 0 \\
Severe irritation & 0 & 0 & 0 & 0 & 0 \\
CH-NC & & & & & \\
Negligible & 100 & 92 & 100 & 100 & 100 \\
Slight irritation & 0 & 8 & 0 & 0 & 0 \\
Moderate irritation & 0 & 0 & 0 & 0 & 0 \\
Severe irritation & 0 & 0 & 0 & 0 & 0 \\
CH-ET-CP & & & & & \\
Negligible & 46 & 8 & 15 & 54 & 62 \\
Slight irritation & 46 & 38 & 69 & 31 & 38 \\
Moderate irritation & 8 & 46 & 8 & 15 & 0 \\
Severe irritation & 0 & 8 & 8 & 0 & 0 \\
CH-ET & & & & & \\
Negligible & 77 & 85 & 92 & 100 & 100 \\
Slight irritation & 23 & 15 & 8 & 0 & 0 \\
Moderate irritation & 0 & 0 & 0 & 0 & 0 \\
Severe irritation & 0 & 0 & 0 & 0 & 0 \\
Commercial formulation & & & & & \\
Negligible & 8 & 0 & 0 & 38 & 92 \\
Slight irritation & 15 & 8 & 38 & 54 & 8 \\
Moderate irritation & 54 & 23 & 62 & 8 & 0 \\
Severe irritation & 23 & 69 & 0 & 0 & 0 \\
\hline Note Numbr represens & & & & 0 \\
\hline
\end{tabular}

Note: Number represents percentages of volunteers who felt slight, moderate, or severe irritation.

Abbreviations: $\mathrm{CH}$, chitosan gel; $\mathrm{NC}$, nanocapsules; $\mathrm{CP}$, capsaicinoids; $\mathrm{ET}$, ethanolic solution.

When the commercial formulation was applied to the skin, the sensation of irritation was higher than with $\mathrm{CH}-\mathrm{NC}-\mathrm{CP}$ or CH-ET-CP. The irritation was considered severe by $23 \%$ and $69 \%$ of the volunteers at 30 and 60 minutes after application, respectively. At these time points, $54 \%$ and $23 \%$, respectively, felt moderate irritation, and only $15 \%$ and $8 \%$ reported slight irritation. At the other time points (90, 120, and 180 minutes), the sensation of skin irritation decreased, with $38 \%, 54 \%$, and $8 \%$, respectively, feeling slight irritation and $62 \%, 8 \%$, and $0 \%$ feeling moderate irritation. At 180 minutes, only $8 \%$ felt slight irritation while $92 \%$ did not feel anything. It was concluded that application of the commercial product led to a more rapid but not persistent sensation of skin irritation, when compared with CH-ET-CP.

After application of the nanostructured formulation (CH-NC-CP), only 23\%, 31\%, and $8 \%$ of the volunteers felt slight irritation after 30,60, and 90 minutes, respectively. After 120 and 180 minutes, $100 \%$ felt negligible irritation. After application of the nanostructured vehicle without capsaicinoids (CH-NC), only $8 \%$ felt slight irritation after 60 minutes, while after 30, 120, and 180 minutes, none of the volunteers described irritation. This indicates that the slight erythema found after application of $\mathrm{CH}-\mathrm{NC}-\mathrm{CP}$ when the visual scale was used is not only due to the presence of chitosan, but probably also due to the action of capsaicinoids on the peripheral nerves.

\section{In vitro skin permeation}

An in vitro skin permeation test was performed in order to study whether the nanoencapsulated capsaicinoids could be released, when in contact with the skin, and permeate through the epidermis, which is the greatest skin barrier, although no or slight skin irritation was observed. The relationship between skin irritation and skin permeation was also investigated. It is well known that capsaicin exerts its analgesic action by depletion in peripheral nerves, including degeneration of epidermal nerve fibers. ${ }^{29}$ Figure 7 shows the cumulative amount of capsaicinoid (capsaicin + dihydrocapsaicin) permeated through an epidermis surface unit as a function of time. Table 3 shows the final cumulative amount permeated after 30 hours, the area under the curve (AUC, calculated using all experimental time points), the flux (calculated using points from 8 to 30 hours, where it was possible to obtain a regression with a correlation coefficient higher than $0.99)$, and the lag time observed for all formulations.

The $\mathrm{AUC}_{0-30}$ values were significantly reduced $(P<0.05)$ when the capsaicinoids were encapsulated within polymeric nanocapsules $\left(40.80 \pm 12.15 \mu \mathrm{g} / \mathrm{cm}^{2} /\right.$ hour) compared to both controls $\left(343.84 \pm 87.54 \mu \mathrm{g} / \mathrm{cm}^{2} /\right.$ hour for CH-ET-CP and $114.92 \pm 16.11 \mu \mathrm{g} / \mathrm{cm}^{2} /$ hour for the commercial product). Regarding the cumulative amount per skin area permeated after

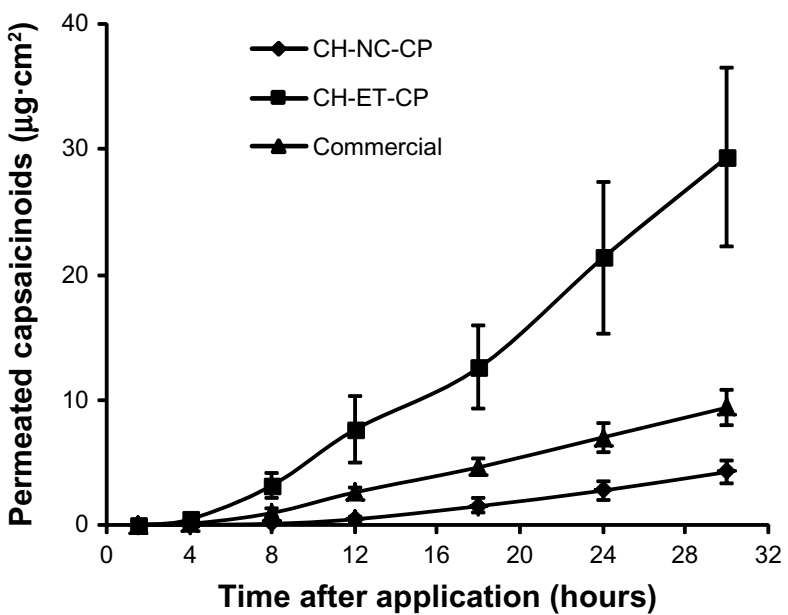

Figure 7 Skin permeation of capsaicinoids incorporated in different semisolid formulations.

Note: Significant differences are described in Table 3.

Abbreviations: $\mathrm{CH}$, chitosan gel; $\mathrm{NC}$, nanocapsules; $\mathrm{CP}$, capsaicinoids; $\mathrm{ET}$, ethanolic solution; Commercial, commercial formulation. 
Table 3 Skin permeation parameters of capsaicinoids from different semi-solid formulations

\begin{tabular}{lcclc}
\hline Formulations & $\begin{array}{l}\mathbf{A U C}_{\mathbf{0 - 3 0}} \\
\left(\mu \mathrm{g} / \mathbf{c m}^{2} / \text { hour }\right)\end{array}$ & $\begin{array}{l}\text { Total amount } \\
\text { permeated }\left(\mu \mathbf{g} / \mathbf{c m}^{2}\right)\end{array}$ & $\begin{array}{l}\text { Lag time } \\
\text { (hours) }\end{array}$ & Flux $\left(\mu \mathrm{g} / \mathbf{c m}^{2} / \mathbf{h o u r}\right)$ \\
\hline $\mathrm{CH}-\mathrm{NC}-\mathrm{CP}$ & $40.80 \pm 12.15^{\mathrm{a}, \mathrm{b}}$ & $4.23 \pm 0.97^{\mathrm{a}}$ & $9.01 \pm 1.04^{\mathrm{a}, \mathrm{b}}$ & $0.19 \pm 0.04^{\mathrm{a}}$ \\
$\mathrm{CH}-\mathrm{ET}-\mathrm{CP}$ & $343.84 \pm 87.54$ & $29.41 \pm 7.17$ & $5.91 \pm 0.69$ & $1.19 \pm 0.29$ \\
Commercial formulation & $114.92 \pm 16.11$ & $9.39 \pm 1.34$ & $5.34 \pm 1.50$ & $0.38 \pm 0.07$ \\
\hline
\end{tabular}

Notes: aSignificant differences $(P<0.05)$ between $\mathrm{CH}-\mathrm{NC}-\mathrm{CP}$ and $\mathrm{CH}-\mathrm{ET}$-CP; bsignificant differences $(P<0.05)$ between $\mathrm{CH}-\mathrm{NC}-\mathrm{CP}$ and commercial formulation. Abbreviations: $\mathrm{CH}$, chitosan gel; $\mathrm{NC}$, nanocapsules; $\mathrm{CP}$, capsaicinoids; $\mathrm{ET}$, ethanolic solution.

30 hours and the flux across the epidermis, a significant difference was observed between the nanostructured formulation $\left(4.23 \pm 0.97 \mu \mathrm{g} / \mathrm{cm}^{2}\right.$ and $0.19 \pm 0.04 \mu \mathrm{g} / \mathrm{cm}^{2} /$ hour, respectively) and the ethanolic formulation $\left(29.41 \pm 7.17 \mu \mathrm{g} / \mathrm{cm}^{2}\right.$ and $1.19 \pm 0.29 \mu \mathrm{g} / \mathrm{cm}^{2} /$ hour, respectively). Regarding the lag times, CH-NC-CP showed significantly higher values $(P<0.05)$ compared with both free drug controls. The differences observed in the permeation parameters for the nanostructured formulation compared with the free drug controls can be explained by the control of drug release due to nanoencapsulation, which has been shown in a previous work. ${ }^{15}$ The rate of drug diffusion from the carrier limits the skin contact. ${ }^{30}$ Before permeating the skin, the capsaicinoids must be released from the nanoparticles, leading to an increase in lag time, a decrease in flux across the skin, and total amount of capsaicinoid permeated after 30 hours. Therefore, by controlling release, the nanoparticles probably prevent a burst release and decrease the amount of substance in contact with the skin tissue at any point in time, possibly leading to a decrease in skin irritation. It is important to note that nanoencapsulation reduced skin permeation, but did not prevent it completely.

Relating permeation to viscosity, it is assumed that low viscosity facilitates release of the drug from the vehicle and, consequently, the degree of skin penetration. The lowest viscosity was observed for CH-NC-CP (16 Pascal seconds) in comparison with the controls (58 Pascal seconds for $\mathrm{CH}-$ ET-CP and 27 Pascal seconds for the commercial product; shear rate 1.76 per second), which could favor permeation of the capsaicinoids when in the nanostructured formulation. However, the nanocapsules played a more important role than the formulation viscosity in modulating skin permeation by the capsaicinoids.

Nanoencapsulation might have modulated the skin permeation in such a way that the sensation of irritation was greatly reduced. The fact that capsaicinoids are still able to cross the skin barrier when nanoencapsulated suggests that the analgesic effect might still occur, since they reach viable epidermis and the epidermal nerve fibers. ${ }^{29}$ In addition, control of skin permeation can lead to sustained permeation, which is interesting when taking into account that 2-4 daily applications are necessary for the analgesic effect of the capsaicinoids. ${ }^{10}$ However, it must be taken into account that the threshold for stimulating the vanilloid receptor may not be achieved when the capsaicinoids are nanoencapsulated, so no effect would be observed. In order to confirm the effect of this promising new formulation, preclinical/clinical trials are needed.

The favorable permeation of capsaicinoids through the epidermis observed when the formulation containing ethanolic solution was applied (CH-ET-CP) is probably explained by the fact that lipophilic drugs, when in a hydrophilic formulation (chitosan gel), tend to leave the vehicle, and come into contact with the skin surface at a faster rate. Further, ethanol at a concentration of $30 \%$ in a CH-ET-CP formulation acts as a permeation enhancer, solubilizing lipids in the stratum corneum..$^{31,32}$

It was previously described that there is a correlation between in vivo physiological responses and in vitro percutaneous penetration of topically applied capsaicin, ${ }^{33}$ which seems to be in agreement with the present work, given that the chitosan hydrogel containing nanoencapsulated capsaicinoids showed less permeation and irritation than the free drug controls. In the work of Shah et al, ${ }^{8}$ the decrease in skin irritation was not related to a decrease in skin permeation of tretinoin. In this case, the authors suggest that the active substance penetrates the skin together with the lipid nanocarriers, so irritation is decreased while skin permeation is not. The penetration of nanoparticles into the skin is more common for lipid nanoparticles but is not commonly observed for polymeric nanoparticles. ${ }^{6}$ In our work, the ability of the nanoparticles to decrease skin irritation is probably explained by the controlled release properties of the nanocarriers.

\section{Conclusion}

This work confirms that the skin irritation caused by application of capsaicinoids can be reduced when polymeric nanocapsules are used to compartmentalize these substances in nanoreservoirs. It is important to note that reduced skin irritation may decrease or even prevent the analgesic effect of capsaicinoids. Therefore, preclinical/clinical trials are necessary in order to confirm the efficacy of such a formulation. In this study, we 
have demonstrated the skin tolerance of an innovative vehicle (polymeric nanocapsules containing chitosan hydrogel), which has good skin adhesion and the potential for enabling controlled skin penetration of encapsulated substances.

\section{Acknowledgments}

The authors thank the Brazilian National Research Council (CNPq), Projects PRONEX and PRONEM from the Foundation for Supporting Research of the State of Rio Grande do Sul (FAPERGS)/CNPq, Brazil-Mexico Bilateral Project CNPq (\#490166/2011-3), Pharmaceutical Nanotecnology Network, Coordination of Improvement of Higher Education Personel (CAPES) for their financial support.

\section{Disclosure}

The authors report no conflicts of interest in this work.

\section{References}

1. Schaffazick SR, Guterres SS, Freitas LL, Pohlmann AR. Physicochemical characterization and stability of the polymeric nanoparticle systems for drug administration. Quím Nova. 2003;26:726-737.

2. Mora-Huertas CE, Fessi H, Elaissari A. Polymer-based nanocapsules for drug delivery. Int J Pharm. 2010;385:113-142.

3. Mohanraj VG, Chen Y. Nanoparticles: a review. Trop J Pharm Res. 2006;5:561-573.

4. Fontana MC, Rezer JF, Coradini K, Leal DB, Beck RC. Improved efficacy in the treatment of contact dermatitis in rats by a dermatological nanomedicine containing clobetasol propionate. Eur J Pharm Biopharm. 2011;79:241-249.

5. Guterres SS, Alves MP, Pohlmann AR. Polymeric nanoparticles, nanospheres and nanocapsules for cutaneous applications. Drug Target Insights. 2007;2:147-157.

6. Contri RV, Fiel LA, Pohlmann AR, Guterres SS, Beck RC. Transport of substances and nanoparticles across the skin and in vitro models to evaluate skin permeation and/or penetration. In: Beck R, Guterres SS, Pohlmann AR, editors. Nanocosmetics and Nanomedicines: New Approaches for Skin Care. Berlin, Germany: Springer; 2011.

7. Castro GA, Coelho AL, Oliveira CA, Mahecha GA, Oréfice RL, Ferreira LA. Formation of ion pairing as an alternative to improve encapsulation and stability and to reduce skin irritation of retinoic acid loaded in solid lipid nanoparticles. Int J Pharm. 2009;38:77-83.

8. Shah KA, Date AA, Joshi MD, Patravale VB. Solid lipid nanoparticles (SLN) of tretinoin: potential in topical delivery. Int J Pharm. 2007;345: 163-171.

9. Pople PV, Singh KK. Targeting tacrolimus to deeper layers of skin with improved safety for treatment of atopic dermatitis. Int J Pharm. 2010;398:165-178.

10. Hayman M, Kam PCA. Capsaicin: a review of its pharmacology and clinical applications. Curr Anaesth Crit Care. 2008;19:338-343.

11. Zi P, Yang XH, Kuang HF, Yang YS, Yu LL. Effect of HP beta CD on solubility and transdermal delivery of capsaicin through rat skin. Int $J$ Pharm. 2008;358:151-158.

12. US Food and Drug Administration. Capsaicin $8 \%$ patch for the management of neuropathic pain associated with human immunodeficiency virus-associated peripheral neuropathy. Available from: http://www.fda. gov/downloads/AdvisoryCommittees/CommitteesMeetingMaterials/ Drugs/AnestheticAndAnalgesicDrugProductsAdvisoryCommittee/ UCM290279.pdf. Accessed November 17, 2013.
13. Barceloux DG. Medical Toxicology of Natural Substances: Foods, Fungi, Medicinal Herbs, Toxic Plants, and Venomous Animals. Hoboken, NJ, USA: John Wiley \& Sons; 2008.

14. Knotkova H, Pappagallo M, Szallasi A. Capsaicin (TRPV1 agonist) therapy for pain relief - farewell or revival? Clin J Pain. 2008;24: 142-154.

15. Contri RV, Kaiser M, Poletto FS, Pohlmann AR, Guterres SS. Simultaneous control of capsaicinoids release from polymeric nanocapsules. J Nanosci Nanotechnol. 2011;11:2398-2406.

16. Contri RV, Katzer T, Pohlmann AR, Guterres SS. Chitosan hydrogel containing capsaicinoids-loaded nanocapsules: an innovative formulation for topical delivery. Soft Materials. 2010;8:370-385.

17. Contri RV, Katzer T, Ourique AF, et al. Combined effect of polymeric nanocapsules and chitosan hydrogel on the effect of capsaicinoids adhesion to the skin surface. J Biomed Nanotechnol. 2014;10: 725-743.

18. Bernkop-Schnürch A, Dünnhaupt $\mathrm{S}$. Chitosan-based drug delivery systems. Eur J Pharm Biopharm. 2012;81:463-469.

19. Wang JJ, Zeng ZW, Xiao, RZ, et al. Recent advances of chitosan nanoparticles as drug carriers. Int J Nanomedicine. 2011;6:765-774.

20. Fessi H, Puisieux F, Devissaguet JP, Ammoury N, Benita S. Nanocapsule formation by interfacial polymer deposition following solvent displacement. Int J Pharm. 1989;55:R1-R4.

21. Santos SS, Lorenzoni A, Ferreira LM, et al. Clotrimazole-loaded Eudragit $^{\circledR}$ RS100 nanocapsules: preparation, characterization and in vitro evaluation of antifungal activity against Candida species. Mater Sci Eng C Mater Biol Appl. 2013;33:1389-1394.

22. Katzer T, Pohlmann AR, Guterres SS, Beck RC. Development and optimization of polymeric nanocapsules intended for ophthalmic administration. Abstract presented at the XII International Macromolecular Colloquium, Gramado, Brazil, September 7-10, 2010.

23. Lin YK, Huang ZR, Zhuo RZ, Fang JY. Combination of calcipotriol and methotrexate in nanostructured lipid carriers for transdermal delivery. Int J Nanomedicine. 2010;5:117-128.

24. Rinaudo M. Chitin and chitosan: properties and applications. Prog Polym Sci. 2006;31:603-632.

25. Schmid-Wendtner MH, Korting HC. The $\mathrm{pH}$ of the skin surface and its impact on the barrier function. Skin Pharmacol Physiol. 2006;19: 296-302.

26. Cross SE, Jiang RY, Benson HA, Roberts MS. Can increasing the viscosity of formulations be used to reduce the human skin penetration of the sunscreen oxybenzone? J Invest Dermatol. 2001;117:147-150.

27. Green BG. Measurement of sensory irritation of the skin. Am J Contact Dermat. 2000;11:170-180.

28. Lee E, An S, Choi D, Moon S, Chang I. Comparison of objective and sensory skin irritations of several cosmetic preservatives. Contact Dermatitis. 2007;56:131-136.

29. Nolano M, Simone DA, Wendelschafer-Crabb G, Johnson T, Hazen E, Kennedy WR. Topical capsaicin in humans: parallel loss of epidermal nerve fibers and pain sensation. Pain. 1999;81:135-145.

30. Yow HN, Wu X, Routh AF, Guy RH. Dye diffusion from microcapsules with different shell thickness into mammalian skin. Eur J Pharm Biopharm. 2009;72:62-68.

31. Lee CK, Uchida T, Kitagawa K, Yagi A, Kim NS, Goto S. Effect of hydrophilic and lipophilic vehicles on skin permeation of tegafur, alclofenac and ibuprofen with or without permeation enhancers. Biol Pharm Bull. 1993;16:1264-1269.

32. Hatanaka T, Katayama K, Koizumi T, Sugibayashi K, Morimoto Y. Time-dependent percutaneous-absorption enhancing effect of ethanol. J Control Release. 1995;33:423-428.

33. Magnusson BM, Koskinen LOD. In vitro percutaneous penetration of topically applied capsaicin in relation to in vivo sensation responses. Int J Pharm. 2000;195:55-62. 


\section{Publish your work in this journal}

The International Journal of Nanomedicine is an international, peerreviewed journal focusing on the application of nanotechnology in diagnostics, therapeutics, and drug delivery systems throughout the biomedical field. This journal is indexed on PubMed Central, MedLine, CAS, SciSearch $\AA$, Current Contents ${ }^{\circledR} /$ Clinical Medicine,

Journal Citation Reports/Science Edition, EMBase, Scopus and the Elsevier Bibliographic databases. The manuscript management system is completely online and includes a very quick and fair peer-review system, which is all easy to use. Visit http://www.dovepress.com/ testimonials.php to read real quotes from published authors.

Submit your manuscript here: http://www.dovepress.com/international-journal-of-nanomedicine-journal 